\title{
Comparing trainers' reports of clicker use to the use of clickers in applied research studies: methodological differences may explain conflicting results
}

\author{
Lynna C. Feng*, Tiffani J. Howell, Pauleen C. Bennett
}

\section{Abstract}

Clicker training refers to an animal training technique, derived from laboratory-based studies of animal learning and behaviour, in which a reward-predicting signal is delivered immediately following performance of a desired behaviour, and is subsequently followed by a reward. While clicker training is popular amongst dog training practitioners, scientific evaluation in applied settings has been largely unsuccessful in replicating the benefits of reward-predicting signals seen in laboratory animal studies. Here we present an analysis of dog trainers' advice and perceptions, conducted to better understand clicker training as it occurs in the dog training industry. Twentyfive sources (13 interviews with dog trainers, 5 websites, and 7 books) were analysed using a deductive content analysis procedure. We found that, for many sources, "clicker training" referred not only to the technique, but also to a philosophy of training that emphasises positive reinforcement and the deliberate application of Learning Theory principles. Many sources reported that clicker training was fun, for both dog and handler, but that it could be frustrating for handlers to learn and sometimes cumbersome to juggle the extra equipment. In addition, while most sources recommended clicker training particularly when training new behaviours, many stated that it was no longer needed once the dog had learned the desired behaviour. When comparing industry recommendations to methods used in applied studies, different criteria were used for predictor signal conditioning. Inadequate conditioning of the predictor signal in empirical evaluations could partly explain the lack of learning benefits in applied studies. While future research is needed to verify the practitioner beliefs in a wider population, these results provide an in-depth description of what clicker training is, at least for the sources analysed, and a potential starting point for understanding methodological factors that could contribute to previous studies' failure to demonstrate the benefits purported to exist by industry practitioners.

Pet Behaviour Science | 2017, Vol.3, 1 - 18

DOI: 10.21071/pbs.v0i3.5786

Lynna C. Feng*, Tiffani J. Howell, Pauleen C. Bennett

School of Psychology and Public Health, La Trobe University.

P.O. Box 199, Bendigo, Victoria 3552

Australia.
Paper Research

* Corresponding author: l.feng@latrobe.edu.au

Keywords:

Clicker training; dog learning; dog trainer perceptions; dog training; learning theory

\section{HIGHLIGHTS}

- Clicker training is both a training technique and a philosophical approach to dog training

- Dog trainers recommend using a clicker-type signal when training novel behaviours but not when rewarding known behaviours

- A mismatch exists between applied and experimental criteria for sufficient signal conditioning

- Empirical studies and individual perceptions disagree on the relative efficacy of clickers versus other signals 


\section{INTRODUCTION}

Clicker training is an animal training technique that employs a clicker (hand-held device that makes a clicking sound when pressed) or other signal to predict the subsequent presentation of a reward (i.e. something the animal wants). Originally introduced to the dog training industry by Karen Pryor in her book called Don't Shoot the Dog! (historical account by Gillaspy et al. 2014; Pryor 1999), clicker training is currently extremely popular. A Google keyword search in August 2016 for the term "clicker training" yielded approximately 1.35 million hits, and Karen Pryor Clicker Training reported over 1400 clicker trainers attending dedicated ClickerExpo seminars in 2016 (Clayton 2016). Somewhat surprisingly, then, a review by Feng et al. (2016) reported that "clicker training" itself is poorly defined and motivations underlying the use of clicker training have yet to be systematically investigated.

Fundamentally, clicker training is derived from laboratory-based studies on Operant Conditioning (Skinner 1938) and the search for mechanisms capable of reducing the adverse effects of delayed reinforcement on learning (e.g. Grice 1948). Laboratory studies suggest that animals learn most quickly when they receive immediate consequences after performing a desired behaviour, but learn more slowly (as reviewed by Lattal 2010) or fail to learn the task at all (Browne et al. 2013) when the consequences are delayed even by a very short interval. The use of a predictor signal, such as the clicker, that predicts the presentation of a subsequent reward, can mitigate the detrimental effects of delayed primary reinforcement (i.e. a time delay between the desired behaviour and reinforcing consequence) in laboratory settings (Lattal 1984). In applied training contexts, delayed reinforcement is often inevitable (e.g. reinforcing behaviour that is performed at a distance). Immediate predictor signals, such as the click of a clicker, have therefore been proposed to mitigate the learning deficit that would normally follow such delays (as reviewed by Feng et al. 2016).

Empirical investigations of the efficacy of clicker training in animal learning typically compare the effect of an auditory reward-predicting signal (such as a clicker) followed by a primary reinforcer with the effect of a primary reinforcer alone (control group). Such studies have been conducted in the following companion animal species: horses (McCall and Burgin 2002; Williams et al. 2004), dwarf goats (Langbein et al. 2007), and dogs (Blandina n.d.; Chiandetti et al. 2016; Smith and Davis 2008). As reviewed by Feng et al. (2016), the findings are surprisingly inconclusive. While goats appeared to learn a shape discrimination task more quickly in a predictor signal treatment group as compared to a control group (Langbein et al. 2007), none of the other studies found the predictor signal treatment condition to be more effective than the treatment used with the control group. In fact, an unpublished thesis reported that dogs in a food-only (control) treatment group reached a higher learning criteria than those in the predictor signal treatment groups (clicker+food and verbal "next" + food) (Blandina n.d.).

Numerous differences across and within the study methodologies, and in comparison with prior laboratory research, could contribute to these seemingly paradoxical findings. To the best of our knowledge, however, behavioural scientists have not yet experimentally evaluated the methodological variations present in these studies. These include: how to introduce the predictor signal, the type of predictor signal used, the task being trained, and the presence and location of a human trainer (as reviewed by Feng et al. 2016). Considering the lack of consistency in methodologies used in empirical studies, the primary aim of this study was to rigorously describe and critically evaluate clicker training methodologies used by or recommended by animal trainers. It was expected that this would provide a better understanding of how clicker training is implemented in applied settings. We discuss this information in the context of available scientific literature and propose additional avenues of research into how methodological differences might influence the efficacy of clicker training protocols.

\section{METHODS}

Advice and perceptions regarding "clicker training" were collected from three sources: interviews with selfidentified dog trainers, bestselling books on clicker training, and clicker training websites. The interviews 
conducted for this project were approved by the La Trobe University Human Research Ethics Committee (approval number: S15-274). All sources were subjected to a content analysis which is described in more detail below.

\section{Interviews with Dog Trainers}

A convenience sample of volunteers $(\mathrm{N}=13 ; 12$ women and one man) were interviewed for this study. All were self-identified dog trainers, fluent in English and ranging in age from $24-73$ years $(\mathrm{M}=41$ years; $\mathrm{SD}=$ 17.59). Recruitment was conducted at a dedicated clicker training conference (Reno, Nevada), online through social media, and via word of mouth until data saturation was reached and no new information was obtained (as suggested by Morse 1995). Each participant completed a brief demographic survey and scheduled an interview at his/her convenience. Semistructured interviews were conducted, in which overall topics included: the participant's experience with clicker training, their beliefs regarding when it is appropriate, and whether it is an effective learning tool. The interview schedule was open ended and participants were encouraged to elaborate on topics they raised. Interviews were held in person, via telephone, or online by web-conference between January-March 2016. All interviews were audio recorded and transcribed for analysis.

Interview participants lived in the United States $(\mathrm{n}=7)$ and Australia $(\mathrm{n}=6)$. All participants self-identified as dog trainers in response to the question "on a scale from 1 (completely disagree) to 5 (completely agree), to what degree do you identify with the statement 'I am a dog trainer'" (completely agree $\mathrm{n}=12$, somewhat agree $\mathrm{n}=1$ ). Although the interview recruitment mentioned clicker training, the advertisement was purposely designed to recruit both trainers who used clickers (hereafter referred to as clicker trainers) and those who did not, in hopes of capturing a range of perspectives. The resulting interviewees were skewed towards identifying as clicker trainers, but the sample included a number of trainers who did not use clickers. In response to the question "on a scale from 1 (completely disagree) to 5 (completely agree), to what degree do you identify with the statement 'I am a clicker trainer'" participants responded as follows: completely agree
$(\mathrm{N}=6)$, somewhat agree $(\mathrm{N}=2)$, neither agree nor disagree $(\mathrm{N}=2)$, somewhat disagree $(\mathrm{N}=2)$, completely disagree $(\mathrm{N}=1)$. For the purpose of having the thoughts and perceptions of trainers who both used and did not use clickers represented in the qualitative analysis, rather than trying to draw comparisons between these individuals and any other sample, this sample was deemed sufficient.

\section{Books about Clicker Training}

The primary objective when choosing books for this review was to examine the most readily available sources of information accessed by everyday dog owners. It was felt that this would be the most objective method of determining predominant advice and practices. As such, a non-personalised Google Books search was conducted with the search terms 'clicker training dog' $^{\prime}$ in November 2015, with the top five results (excluding advertisements) selected for review. The books selected were as follows, in the order they appeared in the Google search:

- Book1: Spector, M. (1999). Clicker training for obedience. Sunshine Books.

- Book2: Pryor, K. (2002). Getting started: Clicker training for dogs. Sunshine Books.

- Book3: Fisher, G. T. (2009). The thinking dog: Crossover to clicker training. Dogwise Publishing.

- Book4: Ray, M. (2008). Click \& train your dog: Using clicker training to transform your common canine into a Superdog. TFH Publications, Inc.

- Book5: Meagher, J. M. (2014). The wonder of clicker training: The complete guide to a nonviolent, positive, compassionate, \& effective way of dog training with clickers. CreateSpace Publishing.

Of these books, three appeared to be guides for dog owners interested in clicker training their own dogs (Book2, Book4, Book5), while the remaining two appeared to be written for individuals, such as trainers, more interested in the nuances of clicker training and why it should be used (Book1, Book3). 


\section{Websites about Clicker Training}

As with choosing books to review, the goal in selecting websites on clicker training was to choose the most readily available sources, believed most likely to reflect industry recommendations received by dog owners. Hence, the top five results (excluding advertisements) from a Google.com query for the term 'clicker training' were selected. The non-personalised search was conducted in March 2016 and the Google.com database algorithm yielded the following results:

- Web1: Karen Pryor clicker training: The leader in positive reinforcement training http://www.clickertraining.com/

- Web2: Clicker training - Wikipedia, the free encyclopedia https://en.wikipedia.org/wiki/Clicker training

- Web3: Clicker training lessons http://www.clickerlessons.com/whatis.htm

- Web4: Clicker training center: Learn to clicker train your dog. http://www.clickertrain.com

- Web5: ClickerSolutions training articles http://www.clickersolutions.com/articles

Similar to the books selected, there appeared to be websites written for both dog owners interested in training their own dogs (Web2, Web4) and those with more in-depth discussions on clicker training practices and controversies, perhaps targeted more towards professional or semi-professional trainers (Web1, Web3, Web5).

\section{Preliminary Analysis}

A cursory glance at the list of books and websites selected for analysis indicates that choosing sources based on search engine results, rather than purposefully selecting a variety of source from different backgrounds, may have resulted in three potential problems. First, all sources focused on basic pet dog training, rather than on training for other activities, such as working or performance roles, where clicker training is very popular (e.g. Gerritsen et al. 2013; Vegh and Bertilsson 2010). Second, there appeared to be a bias towards sources stemming from Karen Pryor Clicker Training. Third, a website entry on clicker training from the online collaborative encyclopaedia Wikipedia was the second highest website search result.

To deal with the first potential problem, it was decided to include two additional books, purposely selected to represent the use of clickers in training dogs for working roles and competitive dog sports. The topranking books in an Amazon.com search of "dog sport clicker training" and "working dog clicker training" sorted by popularity were chosen in December 2015. The books selected appeared to be targeted towards individuals who wanted to become agility dog trainers and service dog trainers, respectively:

- Book6: Bertilsson, E. \& Johnson Vegh, E. (2010). Agility Right from the Start: The Ultimate Training Guide to America's Fastestgrowing Dog Sport. Sunshine Books.

- Book7: Shaw, R. (2015). Service Dog Training - Guide Dogs, Hearing Dogs, Therapy Dogs, Working Dogs, Puppies, Pet Therapy, Emotional Support, Disabled, Clicker Training, Registration, Certification - All Covered. Clovelly Publishing.

While we were cautious about including too much information from Karen Pryor Clicker Training, due to the company's dominant role in the industry, the repeated appearance of associated sources was to be expected. As such, it was decided to proceed as planned. Similarly, while we are aware of the known risks of relying on information from Wikipedia (as discussed by Hilles 2014), it was noted that the opinions presented in the Wikipedia article were not in conflict with those voiced on the other websites. Consequently, we chose to include this website. Since it was ranked second in the Google.com query we conducted, it is likely a leading source of advice on clicker training.

\section{Main Analysis}

To analyse the sources, the deductive qualitative content analysis process described by Elo and Kyngäs (2008) was followed. The purpose was to describe the phenomenon of clicker training within the framework of pre-defined questions. An attempt was made to 
address the following questions: 1) What is clicker training? 2) Why do people use clicker training? and 3) What methods are generally considered 'best practice' in relation to clicker training?

To address these questions, an unconstrained categorisation matrix was developed, where original categories were created based on the overarching questions of interest and a preliminary reading of all sources. Following initial transcription and coding, all coded data from the interviews (Int1-Int13), books (Book1-Book7), and websites (Web1-Web5) were pooled in the hope of generating a broad sense of ideas and perceptions present in the industry. Because this potentially results in some confusion about the source of specific information, we noted where ideas were consistent across source types and, in the analysis reported below, provide the source of sample quotes where appropriate.

During the coding process, sub-categories were created following Elo and Kyngäs' (2008) inductive content analysis approach of creating additional categories that describe the phenomena outside of the pre-determined categories. This allowed for flexibility in expanding on topics based on the information provided by the source materials, while maintaining a focus on the questions of interest. Finally, relevant empirical studies were analysed for the categories that were generated in order to draw methodological comparisons. Based on the analyses, we present a synopsis of the results and, where applicable, discuss the findings within the context of available empirical studies.

\section{RESULTS and DISCUSSION}

\section{What is Clicker Training?}

The first question addressed what came to mind when people thought about the term "clicker training". When defining clicker training, two general aspects were described (Figure 1). Sources defined clicker training in terms of either a training philosophy or a technique. Some even recognised the term as having a dualfaceted nature. Web5 stated: "Clicker training is both a training technique and a training philosophy".

\section{Clicker Training as a Philosophy}

As a philosophy, sources such as Web3 stated that engaging in clicker training meant "using no physical compulsion or corrections whatsoever...using almost entirely positive reinforcement", with an emphasis on applying the "[scientific] principles of learning" (Web5). In addition, sources mentioned the development of a partnership and emphasised a positive experience for both the dog and handler. According to Int9, "it's a partnership that forms...I feel more in tune with the dog". These philosophical aspects of the definition of clicker training were thought to contrast with other popular dog training methods. For example, when comparing clicker training to other methods she had previously

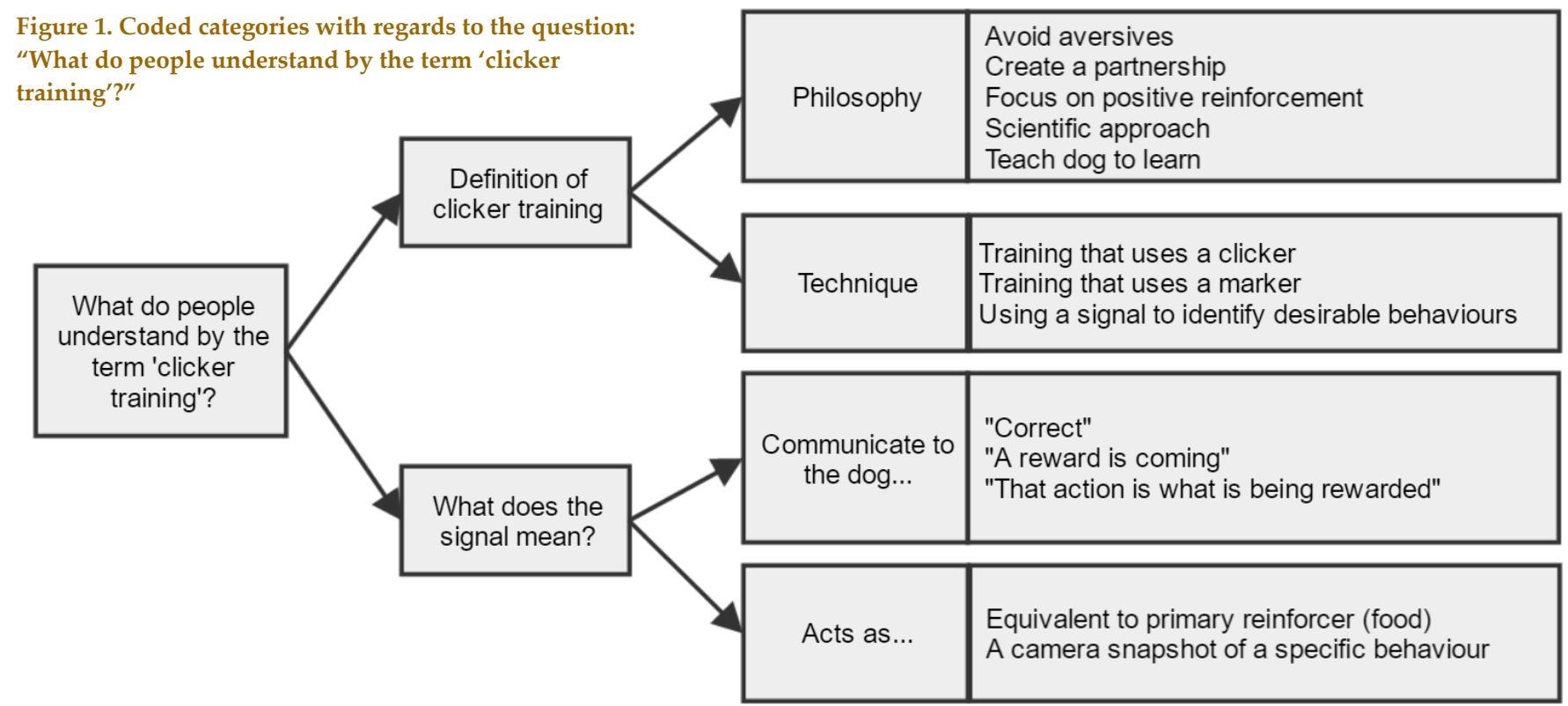


used, Int9 described the following: "It's more... teamwork rather than a dictatorship".

\section{Clicker Training as a Technique}

In terms of the technique of clicker training, most sources described it as a technique employing the use of a clicker to identify desirable behaviours and predict subsequent food rewards. A typical statement was that clicker training is a training method in which the handler "[uses] a 'clicker,' a mechanical device that makes a short, distinct 'click' sound which tells the animal exactly when they're doing the right thing" (Web1). However, some sources indicated that the sound did not necessarily need to be from a clicker device. Instead it could be, as Int4 said, "some [other] kind of marker signal...not always a clicker" such as a spoken word or a whistle, and would still be considered under the umbrella term "clicker training". What appeared to be emphasised was the idea that the signal, whether it was a clicker or otherwise, had to be a form of communication to the dog. Multiple interviewees suggested that, particularly when a dog was first being introduced to the signal, "the click means the food is coming" (Int8), but with practice, the signal begins "[communicating] to the dog that THAT was what I wanted you to do" (Int12).

\section{Dog Perceptions in Clicker Training}

Next, we investigated how people thought dogs perceived the signal. In 2016, the authors reviewed three potential theoretical hypotheses for mechanisms underlying clicker training: Reinforcing, Bridging, and Marking (Feng et al. 2016). The theoretical review found that clicker-type reward-predicting signals most likely function as a secondary reinforcer, but could have bridging and marking properties as well, where the primary function of the clicker-type predictor signal depended on the context in which it was applied. When sources were analysed to investigate industry perceptions of clicker functioning, only a few believed that the clicker functioned as a reinforcer. Int13 stated that "the dog should feel the same way about the 'yes' that it feels about the food." Web5 suggested that, with sufficient pairing, "the click means the same thing to the dog that the presentation of food would." However, not all sources took this position. Int6 stated "I don't think it's a reward." Book5 suggested something similar to the bridging mechanism instead: "[the] clicker serves...to bridge the gap between the time the dog performs the desired behavior and the time they receive the treat."

On the other hand, many sources reported that the more important function of the signal was to "[tell] the animal exactly when they're doing the right thing," as stated by Web1. Similar to the Marking Hypothesis, multiple sources described the clicker as "an event marker" (Web5). However, unlike the marking function described in the scientific literature, where the event marker merely emphasises a point in time without providing reinforcing or punitive feedback, multiple sources believed that the clicker signal communicates to the dog not just "the precise time the dog did the behaviour" (Int10) but that "they're doing the right thing" (Web1) and specifically communicates "what [behaviour] they're being rewarded for" (Int8). For example, instead of using the signal to broadly mean that the dog had done something correct, Int12 said that "giving the clicker sound is like a photograph of the correct behaviour."

Based on these results, it appears that there is not a single, widely accepted consensus on what "clicker training" is. Nor do people agree on what it means (or should mean) to the animal receiving the click. This could potentially explain why it has proved so difficult to demonstrate in applied settings that the clicker is an effective tool. Perhaps those researchers conducting the studies are using the tool in a different way to how it is used in applied settings. Scientific evaluation of clickers in both dogs (Smith and Davis 2008) and horses (Williams et al. 2004) used protocols that tested the clicker for its power as a secondary reinforcer. Williams et al. (2004) found no effect of the clicker as a secondary reinforcer in horses. Likewise, Smith and Davis (2008) also found no difference in rate of task acquisition between clicker and non-clicker groups. However, they did find that dogs rewarded with the click sound alone after performing a behaviour continued to do so for longer than those who received no feedback after performing the behaviour. This suggests that the click was acting as a secondary reinforcer in this context. Given that sources tended to focus more on the communicative effects of the clicker than its power as a reinforcer, it is possible that the context in which 
studies were investigating clicker training differed from how practitioners use it in an applied setting. This could explain the discrepancy between practitioner experiences and empirical results. Regardless, clicker training continues to grow in popularity. The following section reports factors that sources suggest motivate people to use clicker training and those that discourage people from wanting to use clicker training.

\section{Why Do People Clicker Train?}

The second question we aimed to answer was "Why do people clicker train?" To investigate this question, we coded sources based on arguments for and against clicker training relating to the $\mathrm{dog}$, the handler, and the handler-dog relationship (see Figure 2).

Figure 2. Coded categories with regards to the question: "Why do people use clicker training?"

\section{Arguments For Clicker Training}

Consistent with previously published reports of industry perceptions (Smith and Davis 2008), sources suggested that clicker training helps dogs learn faster. However, sources additionally reported numerous other benefits of clicker training for the dog (Figure 2). One unexpected benefit that was reported across multiple sources was that clicker training "encourages [the dog] to think for himself" (Book4) and become a "fully active, thinking participant" (Web1). As Int9 explained, this characteristic of clicker training particularly sets it apart from more traditional training methods where "command compliance" is valued as opposed to nurturing active learning and problem solving skills. Furthermore, sources reported that, with clicker training, dogs appeared eager to learn; Book6 described "a happy and confident dog" and stated that "clicker training will turn your dog into a 'learning junkie,' a dog who is eager to offer behaviors and to experiment to get you to reward." To the best of our knowledge, these

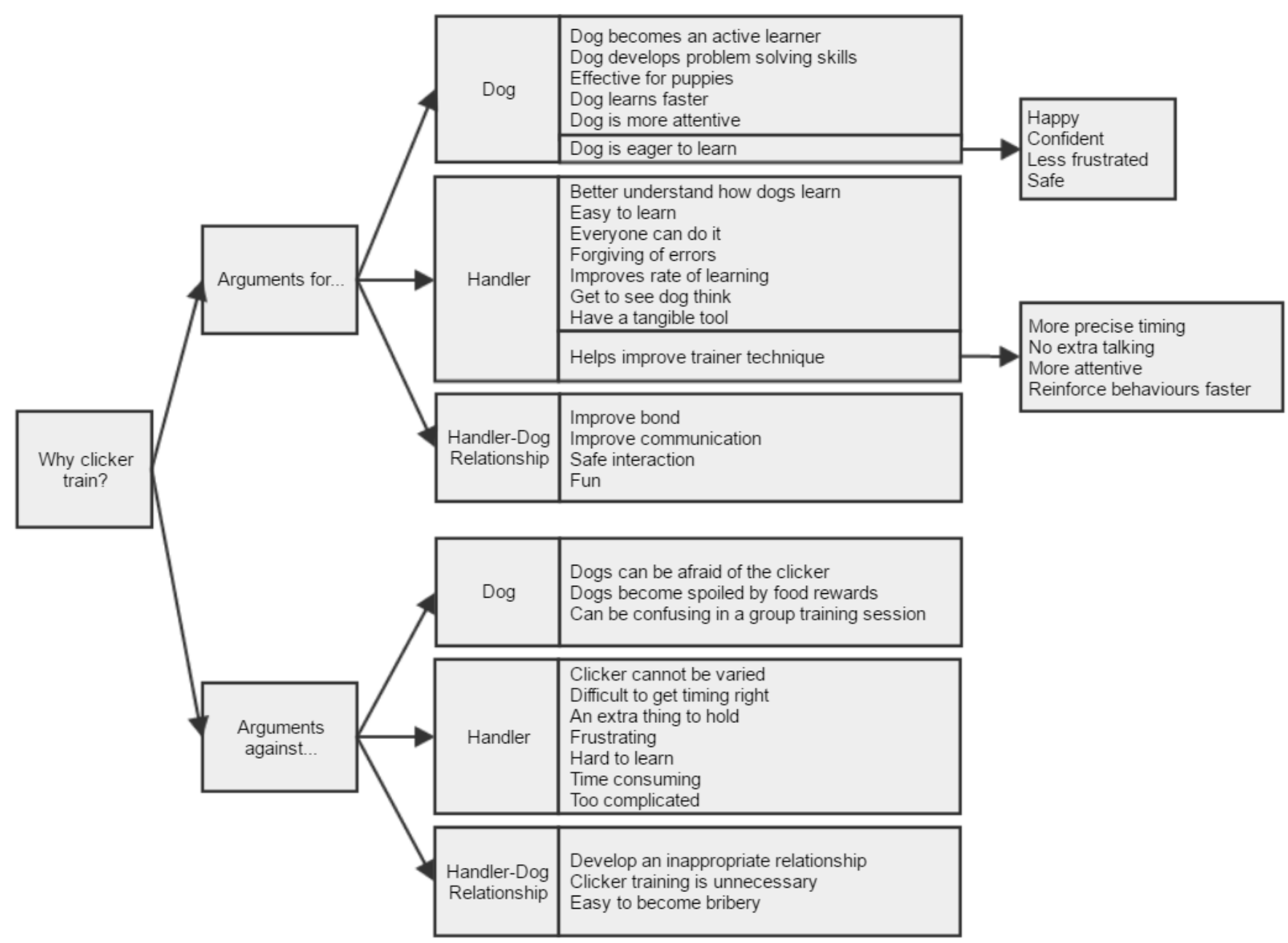


additional benefits of clicker training have not yet been scientifically evaluated, nor have empirical studies been published using dogs or horses who had prior experience with clicker training. Perhaps benefits such as active learning and problem solving develop over time, resulting in more efficient learning after a dog becomes experienced with clicker training. Int9 suggested that "to actually really be a solid clicker training dog would...probably take two months." If so, this could explain why empirical studies, which normally test animals with no previous clicker training experience after a small number of click-treat pairings (20 or fewer in Blandina n.d.; Smith and Davis 2008; Williams et al. 2004; Wood 2007), fail to replicate the enhanced rates of learning reported by clicker training practitioners.

For the most part, sources reported handler benefits that matched those of the dogs. Clicker training was thought to help people learn to train more effectively. Sources attributed this benefit to the focus on precision and timing of reinforcements, and the emphasis on the handler attending to specific criteria in the dog's behaviour during training sessions. In addition, Int4 suggested that clicker training gave handlers an opportunity to "see the little lightbulb" when the dog understood a new task and Int5 said that trainers "[start] to better understand how a dog actually learns." With regards to the handler-dog relationship, multiple sources stated that clicker training "reinforces the bond between dog and trainer" (Book5) and improves communication between dog and handler by providing "a dialogue between trainer and dog" (Book7).

\section{Arguments Against Clicker Training}

Although the sources analysed in this study were heavily biased towards those who support the use of clicker training (all books and websites were on the use of clicker training, and 8 out of the 13 interviewees identified as clicker trainers) there were a number of recurring arguments presented against clicker training. As with the benefits, sources reported costs relating to the dog, handler, and the dog-handler relationship. All reported costs are included in Figure 2. Most commonly, sources stated that clicker training can be time consuming and frustrating for the handler to learn. This came in the form of statements such as: "Clicker training is too difficult for beginners" (Web5), "can...be frustrating" (Int6), and "unless you [have] got an actual [person] to learn from, it is actually quite hard" (Int5). Many people also noted that handlers were often unable or unwilling to carry and handle a clicker device at all times. Int10 described a training situation as follows: "I can't handle it because [I have] a lead in one hand, I've got treats here, the dog's pulling this way and that way and I can't handle it... The clicker is in the way." However, these individuals generally chose to use a verbal signal, such as "yes" or "good," in place of the mechanical click, rather than using no signal at all: "I found it was easier for me to always have my voice on me than to make sure I always have a clicker on me" (Int13). In addition to frustration with learning the mechanical skills, Book3 suggested that, when first learning to use clicker training, handlers might feel "loss of control," which could be related to the coordination required or the less "command-oriented" training philosophy. In light of the reported difficulty and frustration experienced by handlers first learning to clicker train, these consequences should be carefully considered.

With regards to the handler-dog relationship, Int7 voiced concern that using clicker training techniques was "a lot of bribery" and the handler was "putting [themselves] into the position of a servant." Web5 agreed that some "dogs will work only when food or the clicker is present." However, Web5 clarified that this issue happens when "food is misused" and to instead "Reward, don't bribe... The food or toy should be produced only after the dog has performed the behavior." These concerns suggest that clicker training can have negative consequences when used in certain ways. In addition, the concerns presented here have been collected from a sample that predominantly supports the use of clicker training. As such, these reported costs of clicker training are likely not fully representative of the beliefs and experiences of those who do not support the use of clicker training and future research is warranted to better understand these concerns.

\section{Advice and Recommendations}

Many of the arguments against clicker training related to handlers successfully learning to implement clicker training techniques. It is not surprising, then, that sources provided a myriad of rules and suggestions for how clicker training should be implemented (see 
Figure 3). The third question addressed was: "What methods are generally considered 'best practice' in relation to clicker training?" Overall, there were four broad categories of advice offered: 1) How the signal should be introduced, 2) What signals should be used, 3) How the signal should be used, and 4) In what contexts clicker training should be used.

Figure 3. Coded categories for the sub-question: "How should the signal be introduced?" under the main question of "What methods are generally considered 'best practice' with relation to clicker training?"

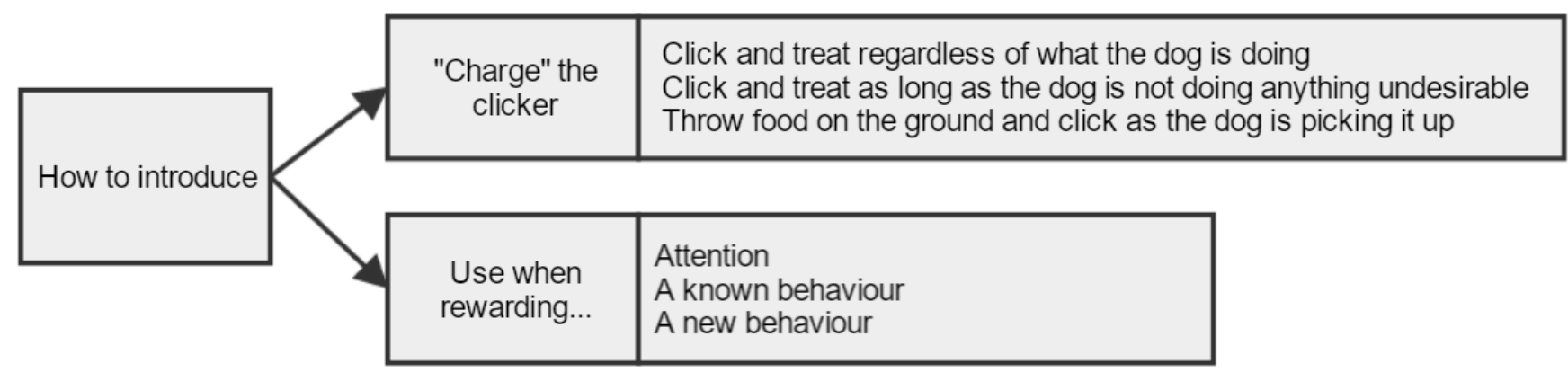

recommendation, but of those who did, recommendations ranged from "two or three times" (Web1) to "one to two hundred reps" (Int13). However, Int13 clarified that 100-200 repetitions was "a gross overestimation" and that dogs seemed to catch on rather quickly, adding that " 3 to 4 can create a classically conditioned response." In fact, a number of sources followed the second method of introducing a new dog to clicker training. These sources suggested that "loading" the clicker by simply pairing the clicker signal with food is unnecessary. Web1 advised to

\section{How the Signal Should Be Introduced}

There were two main methods recommended when introducing a new dog to clicker training: 1) conditioning the signal-food pairing, or 2) skipping the pairing and immediately starting to use the signal when reinforcing desired behaviours (Figure 3). The first method was the more common of the two methods. Sources explained a method called "loading" or "charging" the clicker by which the signal was repeatedly paired with a food reward, essentially building an association between the signal and the subsequent reward. "Create a reflexive link between the sound ('click') and the primary reinforcer ('treat')...The dog learns that click = treat" (Book7). During this "loading" process, sources emphasised that the click-treat is not contingent on the dog performing a specific behaviour, but some clarified that "I'm usually looking for the dog not to be doing a behaviour I don't necessarily want" (Int9) and only click and treat "as long as your dog isn't doing anything naughty at the moment" (Web3).

Interestingly, there was vast variation in the number of pairing presentations that sources recommended for the dog to develop an association between the signal and reward. Many sources did not provide a "immediately choose some specific behavior to click" and Int3 described success without "loading" the clicker: "I just start clicking and treating. I just teach a very very simple behaviour and they very quickly understand what the clicker means." This advice suggests that the "loading" procedure is perhaps unnecessary, but rather that dogs may learn the function of the clicker as their experience increases.

Regardless of the recommended number of repetitions, most sources stated that they had some way of knowing when the dog understood the click-treat association. Book5 explained a common test to determine whether the click-treat association was sufficiently "loaded": "Wait for a time during the day when he is not paying attention to you or he is distracted" then click the clicker and "if he looks at you or comes toward you...he is 'loaded'." Other sources listed behavioural clues such as pairing the click-treat "until the dog begins to look for the treat when he hears the click" (Web4) or "until, when you click they look at you... [with] that expectant face" (Int6). For sources who chose to introduce the clicker within the context of reinforcing a behaviour, a dog was considered to understand the clicker when "at some point, the learner figures it out and begins offering the behavior 'on purpose"' (Web1). 
Somewhat surprisingly, the criteria used in empirical studies for ensuring sufficient click-treat association differ quite considerably from what was mentioned by most of the sources. Smith and Davis (2008) used a sample of clicker-naïve dogs and reported that "[to] ensure that dogs in the clicker group understood that the click predicted food delivery, they were required to meet the criterion of eating the food from the bowl within $5 \mathrm{~s}$ of the click for 10 consecutive trials or to receive a maximum of 20 click-food pairings" (p. 321). This criterion does not exclude the possibility that the dogs merely approached the food once it appeared in the bowl, rather than understanding that the click predicted food delivery. In addition, all dogs, regardless of foodapproaching behaviour, were considered to have been sufficiently conditioned to the click-food pairing after "a maximum of 20 click-food pairings". In this case, industry recommendations appeared more sophisticated, but perhaps less precise, than methods used in empirical studies. Perhaps the precision required by empirical studies has contributed to their failure to demonstrate the efficacy of clicker training, whereas in applied settings individual differences are more easily accommodated.

Figure 4. Coded categories for the sub-question: "What signals can be used?" under the main question of "What methods are generally considered 'best practice' with relation to clicker training?"

\section{What Signals Can Be Used}

The choice of reward-predicting signal appeared to be a contentious issue. A list of all suggested auditory, tactile, and visual signals identified by the interviewees, books and websites considered in this study is presented in Figure 4.

Some sources firmly believed that the mechanical clicker device was the ideal signal, while others thought that a verbal marker would be best. Int9 commented that, "Unlike the verbal or other markers, you have to have a clicker with you. It's just a tool that you have to carry around." Sources who supported the clicker listed traits such as: "precise and unambiguous" (Book6), "[a] clear, unique and consistent sound" (Book5), and "does not convey emotionally loaded approval or disapproval" (Web1), while those who supported a verbal marker listed traits such as: "mouldable as far as what you need at a specific time," "[not] a tool that you have to carry around" (Int13), and "easily available" (Book1). These differences in desirable characteristics of a signal appear to explain individuals' signal of choice. For example, some sources heavily emphasised the importance of handlers being "neutral in their training" (Int13), thus preferring the clicker over a verbal signal for its "non-emotional" (Web5) characteristic. On the surface, this focus on emotional neutrality appears to be in conflict with the fun, partnership-building aspects of clicker training that sources had mentioned as benefits. Yet, Web1 suggests that for handlers, "the word

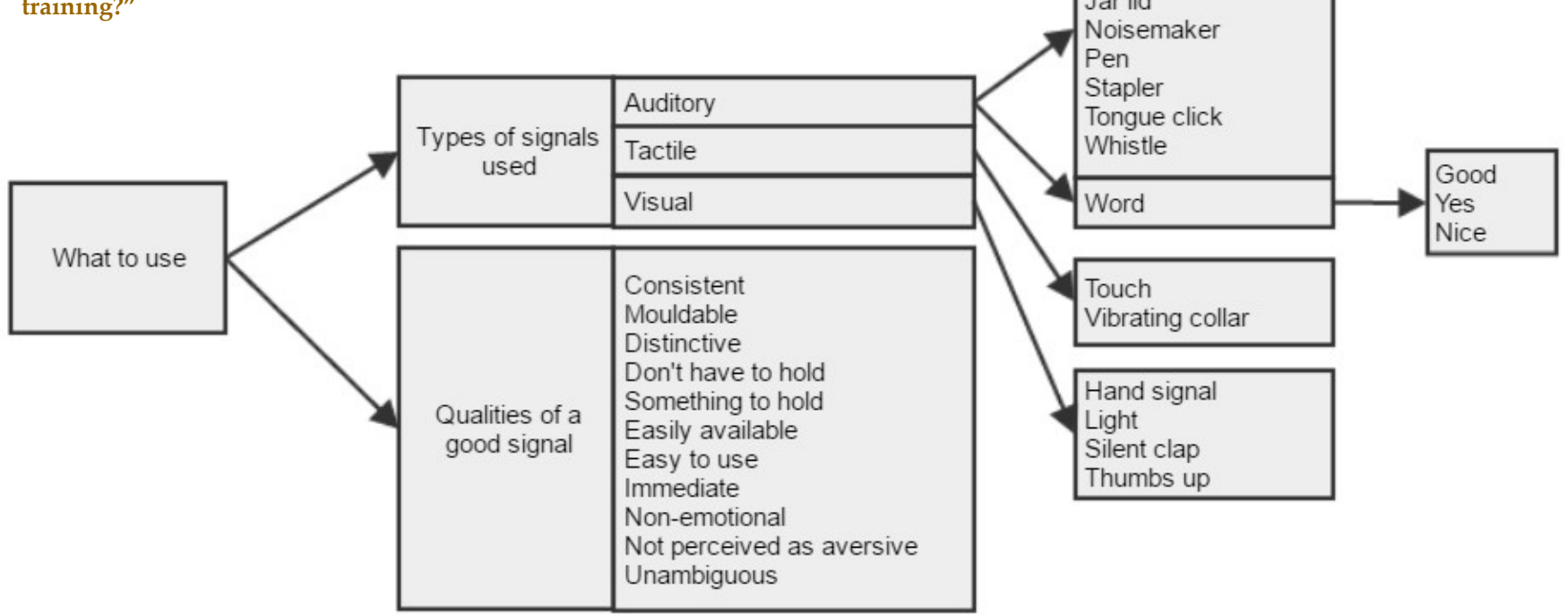


'yes' conveys a sense of social approval...[and] when you can't say 'yes' you may feel frustrated and disappointed, and your posture may actually say 'no!' The dog feels punished -and immediately the learning slows down or stops." Of course, these claims are based on personal experiences rather than empirical evaluation, but undoubtedly warrant further investigation.

One published manuscript and two unpublished Master's theses have compared the efficacy of various signals. Chiandetti et al. (2016) compared a clicker+food treatment group to a verbal "bravo"+food treatment group and found no significant differences between the two groups in dogs' learning or generalizing in a boxopening task. Wood (2007) compared a clicker+food treatment group to a verbal "good"+food treatment group. Unlike Chiandetti et al. (2016), Wood reported that the clicker+food treatment group learned more quickly and required fewer reinforcements compared to the verbal "good"+food group. In Wood's nose-target task, the clicker+food group required an average of 36 minutes to complete the task compared to an average of 59 minutes required for the verbal "good" + food group. Similarly to Chiandetti et al. (2016), Blandina (n.d.) found no significant differences between clicker+food and verbal "next"+food groups with regard to criteria level reached in a shaping protocol; however, both of the groups performed worse than the food-only group. In light of these incongruent results, further investigation is required before empirical studies can provide useful recommendations to practitioners on the relative efficacy of different signals.

\section{How the Signal Should Be Used}

There were three main categories of advice with regards to how the signal should be used: what to click, how to click, and how to reward after the click (Figure $5)$.

In terms of what to click, sources generally agreed that the trainer should pre-define a training criteria ("making sure I have clear criteria" (Int3)) and the signal should be used "[the] exact moment the dog does what you want" (Web3). There was disagreement, however, as to whether the dog should be required to remain in position after the signal in order to receive the subsequent food reward. "[The] click ends the behaviour" (Int8) was a commonly repeated saying. Book2 explained this as follows: "we don't care what the dog is doing when we feed him: only when we click." In contrast, Book5 advised: "if she hears the click and makes a move to come out of her stay, do not give the treat. Rather, lure her back into a sit position, and then give the treat." At present, empirical evidence is unavailable to help determine whether or not choosing to require the dog to maintain the behaviour after the click would impact learning.

With regard to how the signal should be delivered, sources emphasised the importance of delivering the

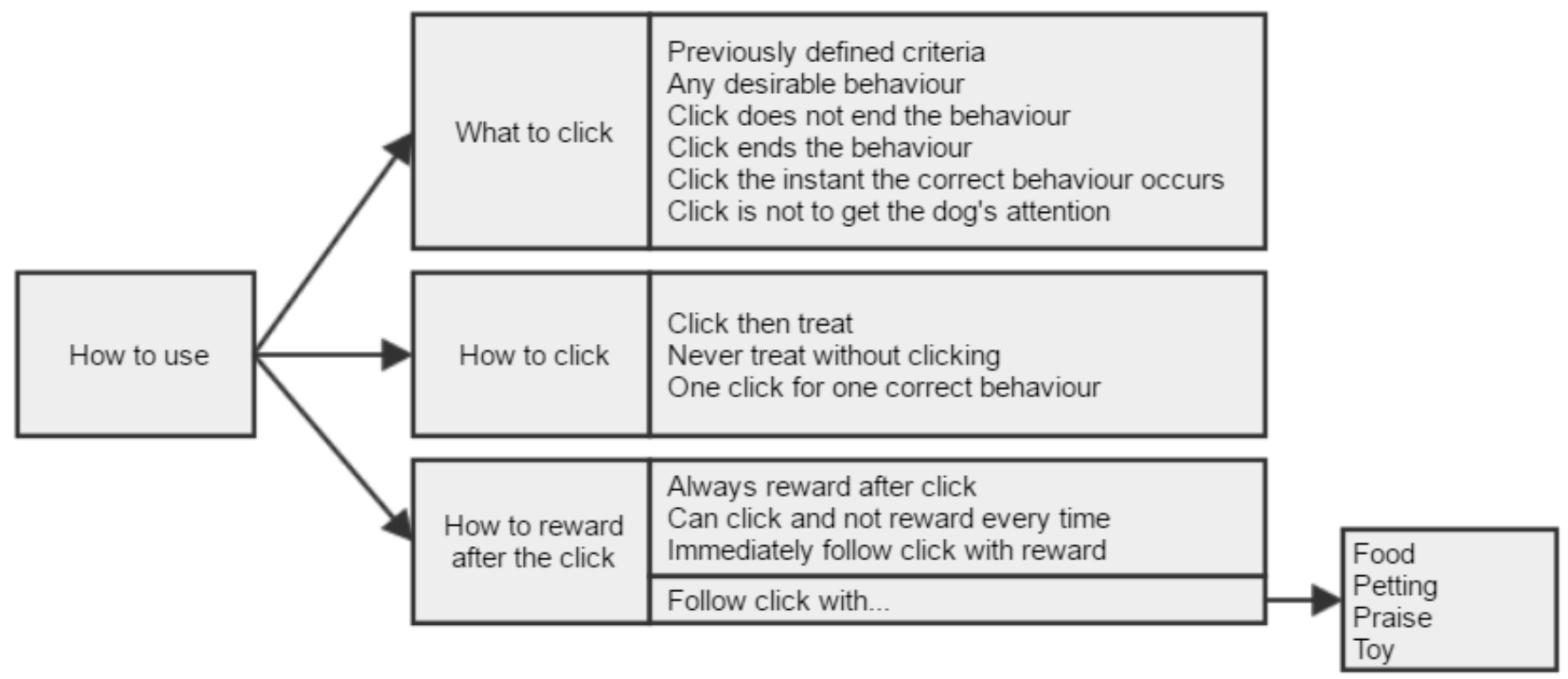

Figure 5. Coded categories for the sub-question: "How should the signal be used?" under the main question of "What methods are generally considered 'best practice' with relation to clicker training?" 
signal prior to the primary reinforcement. "Click first, then treat" (Book5). Sources also emphasised "not clicking multiple times per treat" (Int3); one correct behaviour, regardless of how good or impressive, should only be followed by one click. Most sources also agreed that "you must always reward after a click" (Book5). Int10 explained that she considers the click "as the promise between me and the dog...if I've clicked and don't give them reinforcement you can really see their disappointment." In contrast, a few sources argued that a tangible reward was not required after every signal. For example, Int2 described the following training situation: "'click for this, click for this, click for this' then get the reinforcer at the end."

Numerous studies performed with laboratory animals such as rats (e.g. Zimmerman 1959; Zimmerman and Hanford 1966) have demonstrated that bar-pressing behaviours can be successfully trained and maintained when the secondary reinforcement (equivalent to the click) was only followed by primary reinforcement (e.g. food) intermittently or even when secondary reinforcement was not followed by primary reinforcement at all. However, these subjects were conditioned to the predictor signals under food or water deprivation conditions (e.g. holding at $80 \%$ freefeeding weight or going 23 hours without water). Such deprivation protocols are generally considered unnecessary and unethical for companion animal training (Pryor 1999), making direct comparisons difficult.

Whether or not this or other distinctions between pet dog training conditions and laboratory training conditions affect the function of clicker-type predictor signals is unclear. An unpublished thesis from 2007 began studying the effect of an intermittent ratio of primary reinforcement following a click on learning in dogs. Wennmacher (2007) reported "increased noncompliance and other unwanted behaviors", along with a decreased rate of learning, during a $50 \%$ rate of reinforcement (treat after every other click) as compared to a $100 \%$ rate of reinforcement (treat after every click). These results support the belief that every click needs to be followed by a reward. However, whether this reward needs to be a food reward is unclear. Some sources thought that the reward after the click did not necessarily have to be food and could be praise, petting, or a game of tug instead. Web5 advised to "[give]...food one time, then play with a toy, then just rub his ears and praise him." In fact, in a non-learning context, a recent study using fMRI and preference tests suggested that some dogs prefer social praise over food rewards (Cook et al. 2016). Another study found that dogs preferred petting over praise (Feuerbacher and Wynne 2015). However, when training a nose-targeting response, praise was found to be a relatively ineffective reinforcer compared to food (Feuerbacher and Wynne 2012). Further research is required to determine whether Wennmacher's findings of decreased rates of learning hold true when the click is occasionally followed by a reward other than food.

Surprisingly few sources emphasised the importance of a quickly delivered food reward after the signal. Web1 suggested "delivering the treat as soon as possible after the click," however most interviewees noted that the clicker allowed them to be less strict with the timing of their food reward ("The clicker gives me time to fumble and actually get the actual reward" (Int6)). A 2015 unpublished dissertation, investigating the effect of delayed positive reinforcement on learning in dogs, reported that an immediate signal followed by a onesecond delayed food reinforcement resulted in lower rates of success than immediate food reinforcement. It was also not significantly different from a one-second delayed signal and food (Browne 2015). These results warrant further research, as it is possible that the advice being offered by many practitioners could be inappropriate.

In the only peer-reviewed publication on the efficacy of clicker training compared to food alone with dogs, Smith and Davis (2008) compared a food-only group to a clicker+food group where the food was delivered "approximately 1 s after a click". They found no differences in rate of learning between dogs who received a click+treat versus treat-only. Browne's finding regarding the impact of a one second delay provide one potential explanation for these null results in Smith and Davis (2008), who compared a food-only treatment to an immediate signal, 1 second delayed food treatment. Overall, the available empirical evidence in dogs appears to support the recommendation that the treat should be delivered as soon as possible after the click, and suggest that 
perhaps the clicker does not in fact "give [the trainer] time to fumble and...get the actual reward" (Int6).

\section{Contexts Where Clicker Training Should Be Used}

We asked interviewees if there were particular contexts in which clicker training would be expected to be beneficial and collected a wide range of beliefs. Some

Figure 6. Coded categories for the sub-question: "In what contexts should clicker training be used?" under the main question of "What methods are generally considered 'best practice' with relation to clicker training?" considered clicker training to be appropriate "in all situations" (Int11), while others would only use a clicker-type reward predicting signal "in a controlled environment" (Int7). Others stated that clicker training should be exclusive to professionals: "Don't do it unless you're a professional" (Int1) (Figure 6). The majority of sources fell near the middle and found clicker-type training to be beneficial in some, but not all, contexts. In terms of training contexts where clicker training would not be beneficial, multiple interviewees noted that they "would not use it with BAT®" (Int3) (Behaviour

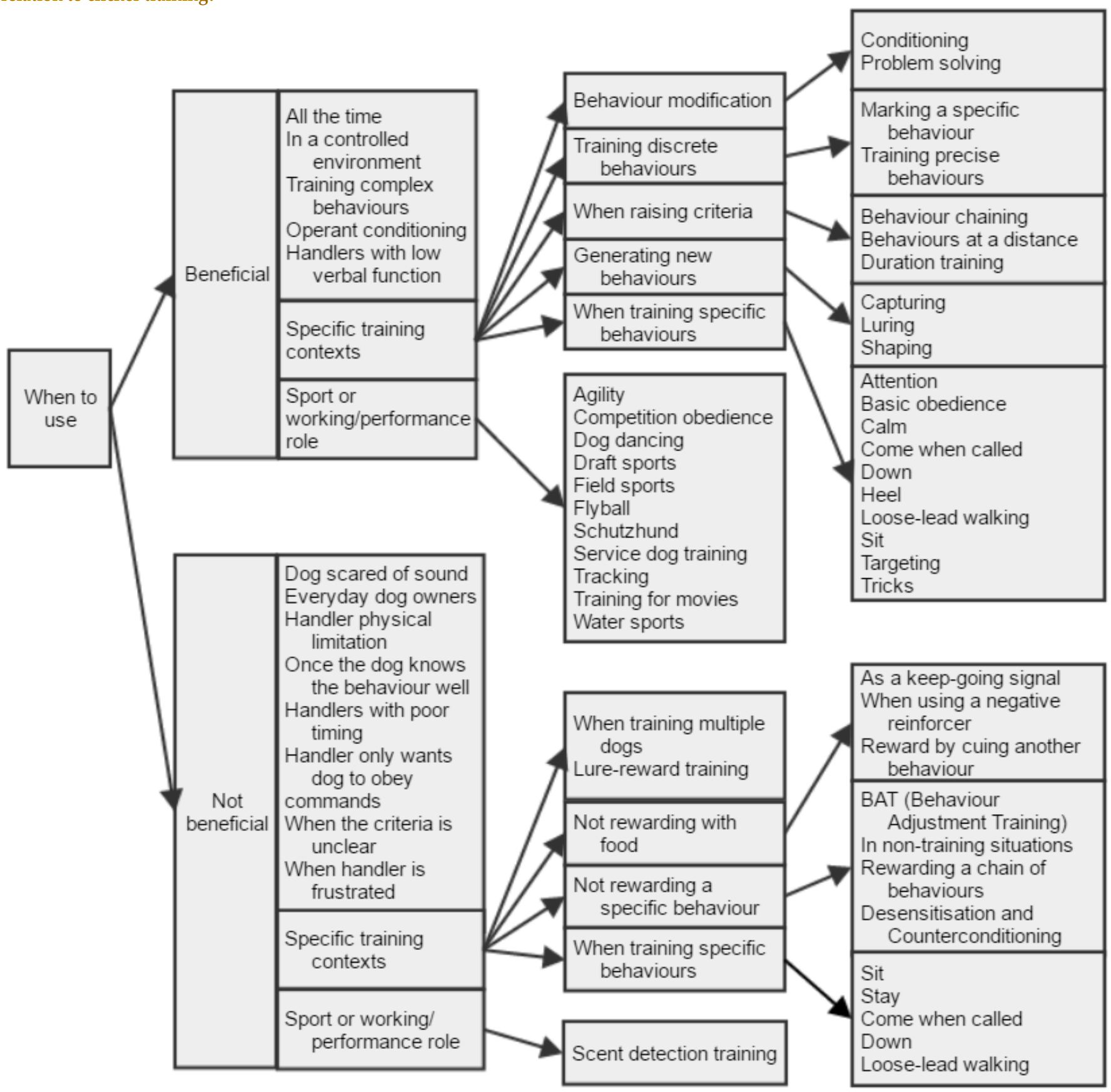


Adjustment Training) or for "scent work" (Int9).

When asked about the contexts in which clicker training was most beneficial, sources listed characteristics such as "activities requiring multiple steps" (Book7) (i.e. taught by shaping), "training behaviours at a distance" (Int13), and "some sort of physical behaviour" (Int1). In addition, across training contexts, most sources emphasised that clicker training was meant to be used when "teaching any new behaviour" (Web5) but "once it's a learned behaviour you don't need [the clicker]" (Int9). In particular, multiple sources recommended clicker training when using a method of behaviour generation called "shaping" (Book2), where new behaviours are taught by systematically reinforcing behaviour approximations closer and closer to a goal behaviour (Yin 2004).

While the efficacy of clicker training in a shaping context has not been assessed relative to a food-only control group, three studies (one unpublished thesis and two peer-reviewed articles) have investigated the efficacy of shaping (combined with clicker training) as compared to other methods of behaviour generation. D'Onofrio's (2015) unpublished thesis investigated the efficacy of shaping as compared to simply capturing spontaneous behaviour and found that, for training the tasks of picking up a wallet or medicine bag, shaping was more effective than just waiting for the behaviour to occur. Fugazza and Miklósi published a pair of manuscripts comparing clicker training (in the context of shaping) to the 'Do as I Do' (Topal et al. 2006) training protocol (Fugazza and Miklósi 2014; 2015). These two studies suggest that, in terms of training time, 'Do as I do' was superior to shaping/clicker training for more complex and object-related actions; however, these differences were less pronounced for simple behaviours and body movement-related tasks. These findings are interesting and potentially suggest a shift toward training techniques that are more cognitively complex than the trial-and-error learning methods historically associated with clicker training. Unfortunately, the extent to which these results can be used to inform judgements regarding the efficacy of the clicker as a reward-predicting signal is limited, as this was not a question directly addressed in the study design.
Strengths, Limitations and Suggestions for Further Research

This study used a range of interviews with dog trainers, and also analysed a number of books and websites, in order to describe and evaluate the phenomenon of clicker training. Such an approach provided a strong foundational understanding of clicker training; however, there are a few limitations that motivate further investigation. Sources were only accessible in English and the majority were from female proponents of clicker training. It is unknown if differences found between sources or source-types are generalisable, and it is possible that not all common opinions have been captured. A larger-scale investigation is required to determine if the results of this study are generalisable to a wider population of dog owners and trainers.

The decision to interview self-identified dog trainers rather than only interviewing those with specific certifications or credentials meant that any individual, including those with no formal training, could have been interviewed. Unfortunately, there does not exist an international governing body for the certification or regulation of dog trainers that could have provided an unbiased means of restricting participants. The qualitative sampling method used for the interviews obtained a diverse sample of individual beliefs existing in the industry, rather than a sample representative of specific groups in a way that would permit generalisations about industry standard beliefs and practices regarding clicker training (Morse 1995). Interviewing individuals with varied dog training backgrounds likely contributed to obtaining a broad and varied perspective.

The use of Google.com database queries allowed the source selection to mimic a likely method by which individuals interested in learning about clicker training might go about locating relevant reading materials. However, this method has a number of potential limitations to its validity and generalisability. First, the database search excludes any sources of advice without an internet presence and could over-represent those sources with better search engine optimisation strategies. Secondly, these sources reflect items that the Google.com proprietary algorithm deemed most relevant and may not reflect the sources most often 
recommended by industry professionals. Finally, this method required the use of keywords specific to clicker training, which resulted in sources that generally recommended its use. As a result, it is unlikely that the content from the sources alone fully represents the beliefs and practices of dog trainers across the industry. Specifically, this method of source selection resulted in limited representation of the thoughts and beliefs of dog trainers who choose not to use clicker training. Nevertheless, that was not the primary objective of this study, and the relative objectivity of this method of source selection compared to alternative methods outweighed these limitations. Evaluation of a wider population of individuals would make it possible to begin determining the relative popularity of certain opinions.

In addition, the use of the Google.com keyword searches did not permit differentiation between sources directed towards dog owners or trainers. It is reasonable to suspect that sources written for a professional audience would include different materials than those written for first-time dog owners. Somewhat fortuitously, however, the results from the search engine query captured both types of sources and, as such, both types of information were represented in the analysis. Ultimately, the use of Google.com to locate clicker training specific books and websites provided a reasonable starting point for understanding the motivations behind clicker training and common "best practice" recommendations. To compare and contrast the opinions of specific groups or source types, future research requires much larger participant numbers and more directed recruitment of particular sample groups, such as those who are for versus against clicker training, or novice dog owners versus experienced dog trainers.

Finally, a number of follow-up studies are recommended based on the findings of this preliminary research. It would be beneficial to test the hypothesis that benefits of clicker training are more pronounced (or perhaps only present) in applied contexts when training dogs that have sufficient prior experience with clicker training. Empirical assessment of the number of click-treat pairings required for pet dogs to develop the association between the clicker sound and subsequent food is also warranted. Furthermore, it would be valuable to know if this click-treat association is required prior to introducing the clicker in a training context. In addition, it would be worthwhile to investigate reported benefits aside from improved rates of task acquisition. For example, the claim that clicker training improves the relationship between the handler and the dog could have important implications by strengthening pet-owner relationships. These and other follow-up studies would begin to elucidate the surprisingly inconclusive results from existing empirical studies, while also informing future methodologies and industry recommendations.

\section{CONCLUSION}

In a recent review, we provided three theoretical accounts of why a device such as a clicker might be expected to enhance learning (Feng et al. 2016). It was noted in this review that empirical studies examining the use of such devices in applied settings had so far produced mixed, but largely unfavourable, results. To begin to explain this paradox, the first aim in this study was to describe and evaluate dog training practitioners' beliefs and perceptions of clicker training relative to empirical studies, so as to better understand the phenomenon as it is practiced in an applied setting. A second aim was to use this information to inform discussion regarding the conflicting evidence on clicker training benefits found both between and within applied and empirical settings. To gather a varied perspective, data were collected from 25 sources across three source types: interviews with dog trainers, popular clicker training books, and websites dedicated to clicker training. These sources were evaluated using a deductive qualitative content analysis process within the framework of three main areas: 1) what clicker training is, 2) why people use clicker training, and 3) 'best practice' clicker training recommendations.

The results of this study provide a comprehensive description of previously unavailable perspectives in the dog training industry with regards to clicker training. Overall, it was evident that many of those practitioners who routinely use clickers or similar devices as training tools, believe strongly that these tools are effective. This may be because clickers are used in practice in different ways than they have been evaluated in available studies. The main findings were 
that: the definition of 'clicker training' is multidimensional; a wide range of beliefs and perceptions exist in regards to why, how, and in what contexts, dog trainers should incorporate clicker-type training methods into their applied practice; and, there is a marked contrast between sophisticated industry tests and seemingly arbitrary empirical criteria for judging when sufficient predictor signal conditioning has taken place.

Each of these factors may contribute to differences in clicker training efficacy seen in practice versus empirical assessments. The multi-dimensionality of clicker training suggests that assessing the clicker as a secondary reinforcer, as has been done in scientific literature (as reviewed by Williams 1994), may not be reflective of what clicker training entails in an applied setting. Likewise, differences in criteria for determining when a dog has had sufficient exposure to the predictor signal for it to function as a learning aid, could have similar implications. These inconsistencies could mean that studies aiming to assess clicker training are not evaluating the phenomenon as used by dog trainers, and as such do not see the same benefits. The varied opinions on how to introduce and use a clicker and the contexts in which they are appropriate may also contribute to differences between practitioner experiences and empirical findings. As such, it is essential that these factors be considered before empirical studies on clicker-type reward predicting signals can confidently be used to inform applied practice. Follow-up studies expanding on these initial results are therefore required to further elucidate the mismatch between industry experiences and empirical evidence.

\section{ACKNOWLEDGEMENTS}

This study was carried out with the support of a La Trobe University Postgraduate Research Scholarship and a La Trobe University Full Fee Research Scholarship.

\section{REFERENCES}

Blandina, A.G. n.d. To click or not to click: Positive reinforcement methods on the acquisition of behavior.
Unpublished thesis, University of Florida.

Browne, C.M. 2015. The effects of delayed positive reinforcement on learning in dogs. Unpublished thesis, University of Waikato.

Browne, C.M., Starkey, N.J., Foster, T.M., McEwan, J.S. 2013. Delayed reinforcement - does it affect learning? Journal of Veterinary Behavior: Clinical Applications and Research 8: e37-e38, doi: 10.1016/j.jveb.2013.04.039.

Chiandetti, C., Avella, S., Fongaro, E., Cerri, F. 2016. Can clicker training facilitate conditioning in dogs? Applied Animal Behaviour Science 184: 109-116, doi: 10.1016/j.applanim.2016.08.006.

Clayton, A. 2016. Opening Session. Lecture notes from ClickerExpo, Reno, Nevada, January 22, 2016.

Cook, P.F., Prichard, A., Spivak, M., Berns, G.S. 2016. Awake canine fMRI predicts dogs' preference for praise versus food. Social Cognitive and Affective Neuroscience, doi: 10.1093/scan/nsw102.

D'Onofrio, J. 2015. Measuring the efficiency of clicker training for service dogs. Unpublished thesis, The Pennsylvania State University.

Elo, S., Kyngäs, H. 2008. The qualitative content analysis process. Journal of Advanced Nursing 62: 107115, doi: 10.1111/j.1365-2648.2007.04569.x.

Feng, L.C., Howell, T.J., Bennett, P.C. 2016. How clicker training works: Comparing reinforcing, marking, and bridging hypotheses. Applied Animal Behaviour Science 181: 34-40, doi: 10.1016/j.applanim.2016.05.012.

Feuerbacher, E.N., Wynne, C.D. 2015. Shut up and pet me! Domestic dogs (Canis lupus familiaris) prefer petting to vocal praise in concurrent and singlealternative choice procedures. Behavioural Processes 110: 47-59, doi: 10.1016/j.beproc.2014.08.019.

Feuerbacher, E.N., Wynne, C.D.L. 2012. Relative efficacy of human social interaction and food as reinforcers for domestic dogs and hand-reared wolves. Journal of the Experimental Analysis of Behavior 98: 105129, doi: 10.1901/jeab.2012.98-105. 
Fugazza, C., Miklósi, Á. 2014. Should old dog trainers learn new tricks? The efficiency of the Do as I do method and shaping/clicker training method to train dogs. Applied Animal Behaviour Science 153: 53-61, doi: 10.1016/j.applanim.2014.01.009.

Fugazza, C., Miklósi, Á. 2015. Social learning in dog training: The effectiveness of the Do as I do method compared to shaping/clicker training. Applied Animal Behaviour Science 171: 146-151, doi: 10.1016/j.applanim.2015.08.033.

Gerritsen, R., Haak, R., Prins, S. 2013. K9 Behavior Basics: A Manual for Proven Success in Operational Service Dog Training. Brush Education.

Gillaspy, J.A., Brinegar, J.L., Bailey, R.E. 2014. Operant psychology makes a splash in marine mammal training (1955-1965). Journal of the History of the Behavioral Sciences 50: 231-248, doi: 10.1002/jhbs.21664.

Grice, G.R. 1948. The relation of secondary reinforcement to delayed reward in visual discrimination learning. Journal of Experimental Psychology 38: 1-16, doi: 10.1037/h0061016.

Hilles, S. 2014. To use or not to use? The credibility of Wikipedia. Public Services Quarterly 10: 245-251, doi: 10.1080/15228959.2014.931204.

Langbein, J., Siebert, K., Nuernberg, G., Manteuffel, G. 2007. The impact of acoustical secondary reinforcement during shape discrimination learning of dwarf goats (Capra hircus). Applied Animal Behaviour Science 103: 3544, doi: 10.1016/j.applanim.2006.04.019.

Lattal, K.A. 1984. Signal functions in delayed reinforcement. Journal of the Experimental Analysis of Behavior 42: 239-253, doi: 10.1901/jeab.1984.42-239.

Lattal, K.A. 2010. Delayed reinforcement of operant behavior. Journal of the Experimental Analysis of Behavior 93: 129-139, doi: 10.1901/jeab.2010.93-129.

McCall, C.A., Burgin, S.E. 2002. Equine utilization of secondary reinforcement during response extinction and acquisition. Applied Animal Behaviour Science 78: 253-262, doi: 10.1016/S0168-1591(02)00109-0.
Morse, J.M. 1995. The Significance of Saturation. Qualitative Health Research 5: 147-149, doi: 10.1177/104973239500500201.

Pryor, K. 1999. Don't shoot the dog! The new art of teaching and training. 2 ed. Bantam Books, New York, New York.

Skinner, B.F. 1938. The behavior of organisms: an experimental analysis. Appleton-Century, Oxford, England.

Smith, S.M., Davis, E.S. 2008. Clicker increases resistance to extinction but does not decrease training time of a simple operant task in domestic dogs (Canis familiaris). Applied Animal Behaviour Science 110: 318329, doi: 10.1016/j.applanim.2007.04.012.

Topal, J., Byrne, R.W., Miklosi, A., Csanyi, V. 2006. Reproducing human actions and action sequences: "Do as I Do!" in a dog. Animal Cognition 9: 355-367, doi: DOI 10.1007/s10071-006-0051-6.

Vegh, E.J., Bertilsson, E. 2010. Agility right from the start: The ultimate training guide to america's fastestgrowing dog sport. Sunshine Books, Inc.

Wennmacher, P.L. 2007. Effects of click+continuous food vs. click+intermittent food on the maintenance of dog behavior. Unpublished thesis, University of North Texas.

Williams, B.A. 1994. Conditioned reinforcement: Experimental and theoretical issues. The Behavior Analyst 17: 261-285, doi:

Williams, J.L., Friend, T.H., Nevill, C.H., Archer, G. 2004. The efficacy of a secondary reinforcer (clicker) during acquisition and extinction of an operant task in horses. Applied Animal Behaviour Science 88: 331-341, doi: 10.1016/j.applanim.2004.03.008.

Wood, L. 2007. Clicker bridging stimulus efficacy. Unpublished thesis, Hunter College, New York, New York.

Yin, S.A. 2004. How to behave so your dog behaves. TFH Publications, c2004. 
Zimmerman, D.W. 1959. Sustained performance in rats based on secondary reinforcement. Journal of Comparative \& Physiological Psychology 52: 353-358, doi: $10.1037 / \mathrm{h} 0045807$.
Zimmerman, J., Hanford, P.V. 1966. Sustaining behavior with conditioned reinforcement as the only responseproduced consequence. Psychological Reports 19: 391401, doi: 10.2466/pr0.1966.19.2.391. 